\title{
A Hierarchical Model for Mobile Ad Hoc Network Performability Assessment
}

\author{
Shuo Zhang ${ }^{1}$, Ning Huang ${ }^{1,2}$ Xiaolei Sun ${ }^{1}$ and Yue Zhang ${ }^{1}$ \\ ${ }^{1}$ School of Reliability and Systems Engineering, Beihang University \\ Beijing 100191, China \\ [e-mail: zhangshuolt@163.com] \\ ${ }^{2}$ Science \& Technology on Reliability \& Environmental Engineering Laboratory \\ Beijing 100191, China \\ [e-mail: hn@buaa.edu.cn] \\ *Corresponding author: Ning Huang \\ Received January 9, 2016; revised March 17, 2016; revised May 17, 2016; accepted June 19, 2016; \\ published August 31, 2016
}

\begin{abstract}
Dynamic topology is one of the main influence factors on network performability. However, it was always ignored by the traditional network performability assessment methods when analyzing large-scale mobile ad hoc networks (MANETs) because of the state explosion problem. In this paper, we address this problem from the perspective of complex network. A two-layer hierarchical modeling approach is proposed for MANETs performability assessment, which can take both the dynamic topology and multi-state nodes into consideration. The lower level is described by Markov reward chains (MRC) to capture the multiple states of the nodes. The upper level is modeled as a small-world network to capture the characteristic path length based on different mobility and propagation models. The hierarchical model can promote the MRC of nodes into a state matrix of the whole network, which can avoid the state explosion in large-scale networks assessment from the perspective of complex network. Through the contrast experiments with OPNET simulation based on specific cases, the method proposed in this paper shows satisfactory performance on accuracy and efficiency.
\end{abstract}

Keywords: Network reliability, MANETs, markov reward chain, multi-states, performance

A preliminary version of this paper appeared in RAMS 2015, USA. In this version, a more realistic node propagation model is introduced to improve the upper model of the hierarchical assessment method. What's more, different node mobility models are considered during the evaluation process. And an efficiency analysis comparison is also presented in the paper. 


\section{Introduction}

$\mathbf{I}_{1}$ Mobile ad hoc networks (MANETs), system performance degrades when optimal paths become unavailable or cooperative nodes are overloaded. In those cases, network connectivity reliability is still maintained at a high level, but the success probability of MANETs missions may be influenced by the performance degradation. Thus, it is necessary to combine performance parameters into MANETs reliability assessment. Performability which means the system's ability to perform when performance degrades as a consequence of faults is very important to MANETs [1-4].

In recent years, many studies have been focused on the performability of MANETs. There are mainly three ways for the performability assessment of MANETs: (1) Extensions of traditional network reliability methods [5-11], (2) Analyzing the performance of the MANETs based on the complex network theory [12-16], and (3) Evaluating the performability of MANETs using network simulation software [17-23].

Many traditional reliability methods, such as Dynamic Fault Free (DFT) and Dynamic Reliability Block Diagram (DRBD), were improved to analyze the performability of MANETs. Distefano et al. [5] used DFT and DRBD to build the dependability models, which can be transformed into Petri Nets. In Ref [6], an integrated DFT and Monte Carlo method was proposed. DFT and DRBD methods can be used to evaluate the reliability of static topology network. However, they have many disadvantages on dynamic topology modeling. The similar problem also exists in the Markov Chain model. Through Markov Chain models, we can accurately analyze the performability of nodes and small scale networks, but the complexity increases awesomely when the scale of network becomes larger. Cello et al. [11] used Continuous-time Markov Chains (CTMC) to analyze the connectivity and average packet delivery delay of the Intermittently-Connected Networks (ICNs), but the topology of the case was very simple. Azni et al. [7] used the Semi Markov model to evaluate the performability of MANETs and took the correlated behavior of nodes into consideration. The effect of correlated failures was described clearly, but when it was applied to a large MANETs, the state explosion problem emerged. In addition to the dynamic topology, the wireless features of MANETs also bring some challenges and some studies have been accomplished to overcome these challenges. The ways of data transfer were taken into consideration in Ref [8] and Groenevelt et al. [9] presented a more accurately stochastic model of the message delay in MANETs, which had only two input parameters: the number of nodes and the parameter of an exponential distribution which describes the time until two random mobiles come within communication range of one another. The model was extended in Ref [10] for delay evaluating of opportunistic forwarding MANETs under heterogeneous mobility. However, these methods still can't cope with the state explosion of large-scale MANETs.

Complex network theory is widely employed in large-scale network analysis. Many studies analyzed the performance and reliability of MANETs from the perspective of complex network theory. Applying small-world networks in wireless networks was first 
investigated by Helmy et al. [12], in which simulation results were used to study the behavior of wireless networks as a result of random addition of distance limited short cuts. Further, Banerjee et al. [13] analyzed the self-organization in MANETs as small worlds while maintaining connectivity. Complex network theory can analyze the whole network characteristic, such as the mobility and propagation models, of big scale MANETs. Hossmann et al. [14] analyzed the performance of human mobility and Panisson et al. [15] evaluated the dynamic of human proximity data spreading in MANETs. But the performance degradation of nodes was not considered in these researches. Rezende et al. [16] validated the complex networks properties of MANETs and analyzed the impact of mobility based on characteristics of small-world networks, but the influence factors of the model were too simple and the multi-states of nodes were also ignored. All in all, this kind of method is not good at failure modeling and it is hard to evaluate the multi-states of nodes performance.

Network simulation software such as NS-2, OMNET++ and OPNET can simulate the network behaviors accurately. Many related researches have been conducted to analyze the performance of MANETs using these simulation softwares. In Ref [17], the authors used NS-2 to evaluate the performance of MANETs under single-path and multi-path routing. NS-2 was also used to analyze the performance of MANETs on demand distance vector routing in Ref [18]. In Ref [19], performance analysis of random-based mobility models in MANETs was accomplished based on OMNET++. In Ref [20], delay and throughput evaluation of Vehicular Ad-hoc Networks (VANETs) was presented. In Ref [22], a method to compare performance of different routing protocols is proposed. Garg et al. [21] improved this method and presented a comparative performance analysis of temporally ordered routing algorithm (TORA) and Dynamic Source Routing (DSR). In Ref [23], a multipath routing protocol called MPOLSR \& MDART was proposed, and the performance of table driven multipath routing protocols in MANETs under different scenarios were analyzed based on NS-2. This kind of simulation method is always used to analyze the performance of a specific routing protocol, but it is not good at failure modeling and the earlier period development is complex. What's more, when facing the large-scale networks, the efficiency of this method is unsatisfactory.

In this paper, we propose a hierarchical model for performability analysis of MANETs with repairable components. The upper level of the model describes the characteristics of the network, such as the mobility and the propagation. The Markov reward chain is used to describe the performance failures of the nodes in the lower level. We bring the Markov Chain and complex networks theory together, because the former has its unique advantages on fault modeling and the latter is generally used to analyzing the performance of large-scale network systems. When analyzing the performability of a MANETs, we transform it into a specific multi-phased system based on its mission profile and then calculate the characteristic path length of each phase at the upper level. Based on the Markov reward chains at the lower level and the characteristic path length, the probability matrix of each single phase can be calculated. At last, the performability of the MANETs can be gotten from the product of the probability matrixes. A specific case of MANETs is presented to support our approach in this paper. What's more, a more realistic node 
propagation model can be introduced to improve our assessment model. Then, accuracy verification is carried out by comparing the result between OPNET simulation experiment and our hierarchical model. .At last, an efficiency analysis is also presented, which shows that the hierarchical model has a good performance in efficiency.

The remainder of the paper is organized as follows. Section 2 presents our hierarchical assessment method, and explains the evaluation process. In section 3, we use the hierarchical method to analyze the performability of a classic MANETs case, and we improve our hierarchical assessment method by modifying the propagation model in upper model. Also different nodes mobility models is taken into consideration during the simulation. In section 4, we present contrast experiments with OPENT simulation to test the accuracy and efficiency of the hierarchical assessment method.

\section{Hierarchical Assessment Method}

Dynamic topology and the multi-states of nodes performance degradation are both the main influence factors of MANET performability. The former is a feature from the macroscopic angle of the network and the latter is a microscopic characteristic. In order to get a more authentic result, it is necessary to combine the dynamic topology and multi-states of nodes together in performability analyzing of MANETs. The hierarchical model proposed in this paper captures the multi-states of nodes at the lower level based on MRC model, and evaluates the dynamic topology at the upper level from the perspective of complex network.

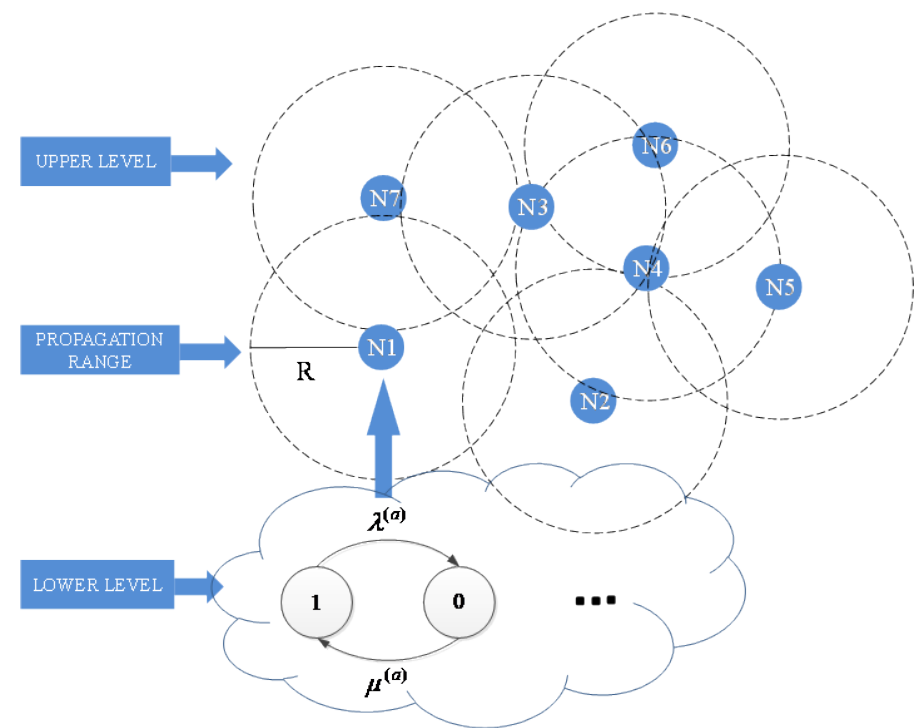

Fig. 1. Hierarchical performability model.

In a MANETs $G=\{V, E\}$, let $V=\left\{v_{1}, v_{2}, \cdots, v_{n}\right\}$ denote the set of $N$ nodes in the network and $E=\left\{\boldsymbol{e}_{1}, \boldsymbol{e}_{2}, \cdots, \boldsymbol{e}_{\boldsymbol{m}}\right\}$ denote the set of $M$ edges in the network. At the lower 
level of the hierarchical model, each node $v_{i}$ can have its own MRC model, which can provide the performance states of nodes for the upper level model to judge whether the communication between two nodes is successful when they are in the communication range. The upper level model is modeled as a small-world network to capture the characteristic path length of the network, which means the average minimum edges between each two nodes in the network [16]. Because of the dynamic topology, the number of edges in $E$ changes as the network working. The characteristic path length promotes the MRC of nodes from the lower level model into a state matrix of the whole network, which can be used to obtain the performability of the network through some further calculation.

In the rest part of this section, a detailed description of the hierarchical performability assessment method is proposed in three sub-sections. We show the lower level of the model, which presents the MRC model of the nodes performance, first and then the characteristic path length in the upper level of the model is presented, which decides the efficiency of the probability matrix from the MRC models into performability calculation. Finally, the assessment method of system performability is discussed.

\subsection{Node States Model}

Network performability is the system's ability to perform when performance degrades as a consequence of faults. Delay is chosen as the main indicator in this paper, because users usually care more about this performance in MANETs [24-26]. In Ref. [24], the expected excess delay in overload (EEDO) is taken as the survivability performance measure for a wireless ad-hoc network. Kaur [25] studied the performance of Mobile Ad-hoc network to analyze the impact of different routing protocols and the performance is analyzed using throughput, delay and media access delay parameters. Ravinder [26] evaluated the performance of AODV and DSR routing protocol under wormhole attack. Performance parameters are Average end to end delay, Throughput, and Packet delivery ratio.At the lower level, we model each node $c$ in the network as an arbitrary finite state and obtain the homogeneous MRC with state space $\left\{1,2, \ldots, k^{(c)}, \ldots, n^{(c)}\right\}$. For simplicity, we assume states $\left\{1,2, \ldots, k^{(c)}\right\}$ are operational states and $\left\{k^{(c)}+1, k^{(c)}+2, \ldots, n^{(c)}\right\}$ are down states. And $\left\{d_{1}^{(c)}, d_{2}^{(c)}, \ldots, d_{m}^{(c)}\right\}$ are the delay values of the operational states, each of which has its own delay value. When analyzing the performability of MANETs, the network is considered as a multi-phased system [27]. The nodes have different probability state vector in each phase. Details of the states of $c$ and state transitions are described as follows:

$A^{(c)}$ (generator matrix for the availability model of component $c$ ) can be written in the partitioned form as (1).

$$
A^{(c)}=\left(\begin{array}{ll}
A_{11} & A_{12} \\
A_{21} & A_{22}
\end{array}\right)_{n^{(c)} \times n^{(c)}}
$$


Where $A_{11}$ is a $k^{(c)} \times k^{(c)}$ matrix and contains transition rates from operational state to operational state. Similarly, $A_{21}, A_{12}$, and $A_{22}$ contain the transition rates from failed state to operational state, operational state to failed state, and failed state to failed state, respectively.

$R^{\text {(c) }}$ (generator matrix for the reliability model of component $c$ ) can be written in the partitioned form as (2).

$$
R^{(c)}=\left(\begin{array}{cc}
A_{11} & A_{12} \\
0 & 0
\end{array}\right)_{n^{(c)} \times n^{(c)}}
$$

\subsection{Characteristic Path Length}

We combine a MRC performance model with the small-world network theory to analyze the performability of MANETs. In the upper level of the hierarchical model, the characteristic path length is used to represent the macroscopic features of the network. The dynamic topology, which may be caused by many MANETs features, such as different mobility and propagation models, can be reflected by the characteristic path length. We can obtain the characteristic path length by (3).

$$
y=2\left(\sum_{i=1}^{i=n-1} \sum_{j=i+1}^{n} l_{i j}\right) /(n(n-1))
$$

Where $n$ is the scale of the MANETs and $l_{i j}$ means the minimum path length between node $i$ and node $j$. Then, the minimum path length between each node pairs should be obtained firstly to calculate the characteristic path length. For example, for Fig. 1, we calculate the matrix of the minimum edges between each node pairs and show the results in Table 1.

Table 1. Minimum edges between two nodes in Fig. 1.

\begin{tabular}{|c|c|c|c|c|c|c|c|}
\hline & $\mathrm{N} 1$ & $\mathrm{~N} 2$ & $\mathrm{~N} 3$ & $\mathrm{~N} 4$ & $\mathrm{~N} 5$ & $\mathrm{~N} 6$ & $\mathrm{~N} 7$ \\
\hline $\mathrm{N} 1$ & & 4 & 2 & 3 & 4 & 3 & 1 \\
\hline N2 & 4 & & 2 & 1 & 2 & 2 & 3 \\
\hline N3 & 2 & 2 & & 1 & 2 & 1 & 1 \\
\hline N4 & 3 & 1 & 1 & & 1 & 1 & 2 \\
\hline N5 & 4 & 2 & 2 & 1 & & 2 & 3 \\
\hline N6 & 3 & 2 & 1 & 1 & 2 & & 2 \\
\hline N7 & 1 & 3 & 1 & 2 & 3 & 2 & \\
\hline
\end{tabular}

The number in line $i$ and column $j$ represents the minimum length between the nodes $(i$, 
$j$ ). For example, between the node $N_{1}$ and $N_{4}$, the minimum path between them is $N_{1}-N_{7}-N_{3}-N_{4}$. Because $N_{2}$ and $N_{4}$ are not in the communication range of $N_{1}$, there is no shorter path between $N_{1}$ and $N_{4}$. Therefore, communication between the two nodes at least through three hops, the number in line 1 and column 4 is 3 .

Then, we can obtain the characteristic path length by formula (3). That is, the characteristic path length of the network in Fig. 1 is 2.

When the scale of the network becomes larger, the breadth-first search (BFS) algorithm can be used to obtain the minimum edges between nodes.

\subsection{System Performability}

If the probability vector for node $c$ at the beginning of phase $i$ is $v_{i-1}^{(c)}$, then we can obtain the probability vector for staying in operational states during phase $i$ by (4).

$$
u_{i}^{(c)}=v_{i-1}^{(c)} e^{R^{(c)} T_{i}}\left(\begin{array}{cc}
I_{k^{(c)} \times k^{(c)}} & 0 \\
0 & 0
\end{array}\right)
$$

Where $T_{i}$ is the mission time, $I$ is a $k^{(c)} \times k^{(c)}$ unite matrix, and the $x_{t h}$ element of $u_{i}^{(c)}$ represents the probability that $c$ remains operational throughout phase $i$, and arouse delay $d_{x}^{(c)}$. Suppose the characteristic path length of the network in that phase is $y$, and the failure criterion is $f$, then the performability of the network at phase $i$ can be obtained by (5).

$$
P_{i}=\sum P\left(\left(d_{s_{1}}^{(1)}+\cdots+d_{s_{j}}^{(j)}+\cdots+d_{s_{y}}^{(y)}\right)<f\right)
$$

Where $0<s_{j}<k$ and $d_{s_{j}}^{(j)}$ represents the delay caused by the $j_{t h}$ transmission node when it is in state $s_{j} . P_{i}$ represents the ability that the MANETs can remain a good delay performance as requested through phase $i$. Hence, we can obtain the network's performability of each phase through the whole mission time. The method is applied to a classic case in the next chapter.

\section{Case Study and Analysis}

In this section, we use the hierarchical model to evaluate the performability of a specific case extended from the Thales Research and Technology Report (UK) [28]. Firstly, the initial parameters of the case and the process to assess its performability using the method proposed above are presented. Secondly, a more realistic radio propagation model is introduced to modeling the link reliability in our assessment method. And the network performance is comparative analyzed when using different radio propagation models. Finally, a different mobility model is also added in this case to compare with the nodes random mobility. 


\subsection{Case Introduction and Performability Assessment}

The test network has 90 nodes, all of which follow random direction mobility model and free-space propagation model. The specific parameters of the network are shown in Table 2.

Table 2. Parameter values of the case.

\begin{tabular}{|c|c|}
\hline Parameters & Value \\
\hline Network size & $1000 \mathrm{~m} * 1000 \mathrm{~m}$ \\
\hline Node amount & 90 \\
\hline Transmission distance & $150 \mathrm{~m}$ \\
\hline Mobile model & Random direction \\
\hline Propagation model & Free-space (FS) \\
\hline Node delay & $(0.0,0.001,0.002) \mathrm{ms}$ \\
\hline Failure criterion & $2.5 \mathrm{~ms}$ \\
\hline Failure rate & 0.005 \\
\hline Repair rate & 0.998 \\
\hline Simulation duration & $1 \mathrm{~h}$ \\
\hline
\end{tabular}

Four distinct topologies of the network at different moments are shown in Fig. 2. As the nodes move around, the value of the characteristic path length changes in different simulation phase.
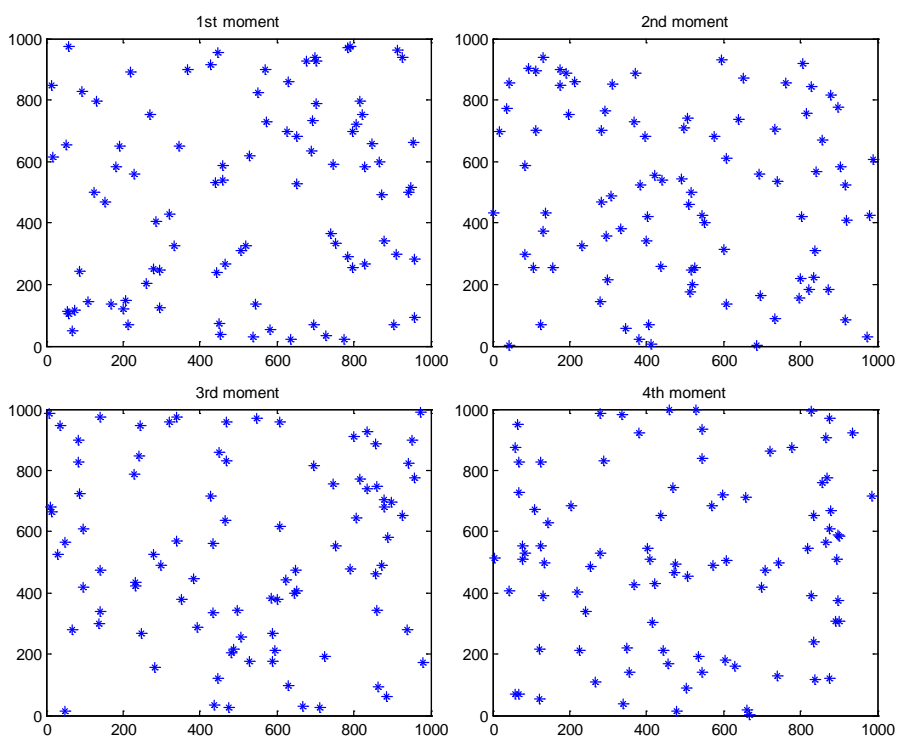

Fig. 2. The upper level model of the test network. 
In the lower level of the model, we suppose that each node has a hot backup. Thus, there are three node performance states through the whole mission of the network. The MRC model of the nodes in this network is shown in Fig. 3.

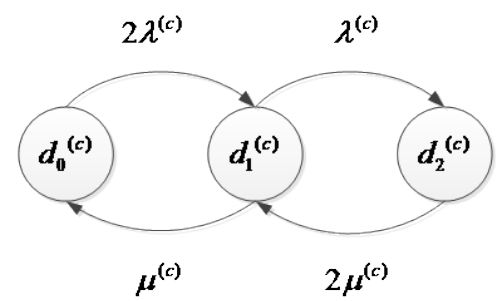

Fig. 3. The MRC model of nodes.

Where $\boldsymbol{\lambda}^{(c)}$ is the failure rate, $\boldsymbol{\mu}^{(\boldsymbol{c})}$ is the repair rate and $\boldsymbol{d}_{\boldsymbol{k}}^{(c)}$ represents the delay caused by node $c$ when it is in the $k_{t h}$ state. According to the Markov chain in Fig. 3, we can obtain the states transition matrixes as (6) and (7).

$$
\begin{array}{r}
A^{(c)}=1\left[\begin{array}{ccc}
-2 \lambda^{(c)} & 2 \lambda^{(c)} & 0 \\
\mu^{(c)} & -\left(\mu^{(c)}+\lambda^{(c)}\right) & \lambda^{(c)} \\
0 & 2 \mu^{(c)} & -2 \mu^{(c)}
\end{array}\right] \\
R^{(c)}=1\left[\begin{array}{ccc}
-2 \lambda^{(c)} & 2 \lambda^{(c)} & 0 \\
\mu^{(c)} & -\left(\mu^{(c)}+\lambda^{(c)}\right) & \lambda^{(c)} \\
0 & 0 & 0
\end{array}\right]
\end{array}
$$

According to performability assessment model based on the formula (4), (5), (6) and (7), and the parameters in Table 2, the network performability can be obtained. The result is shown in Fig. 4, where y-axis represents the performability of the network and $\mathrm{x}$-axis represents the 100 phases of the whole network mission. The duration of each phase is 36 seconds, and the whole simulation time is 1 hour. 


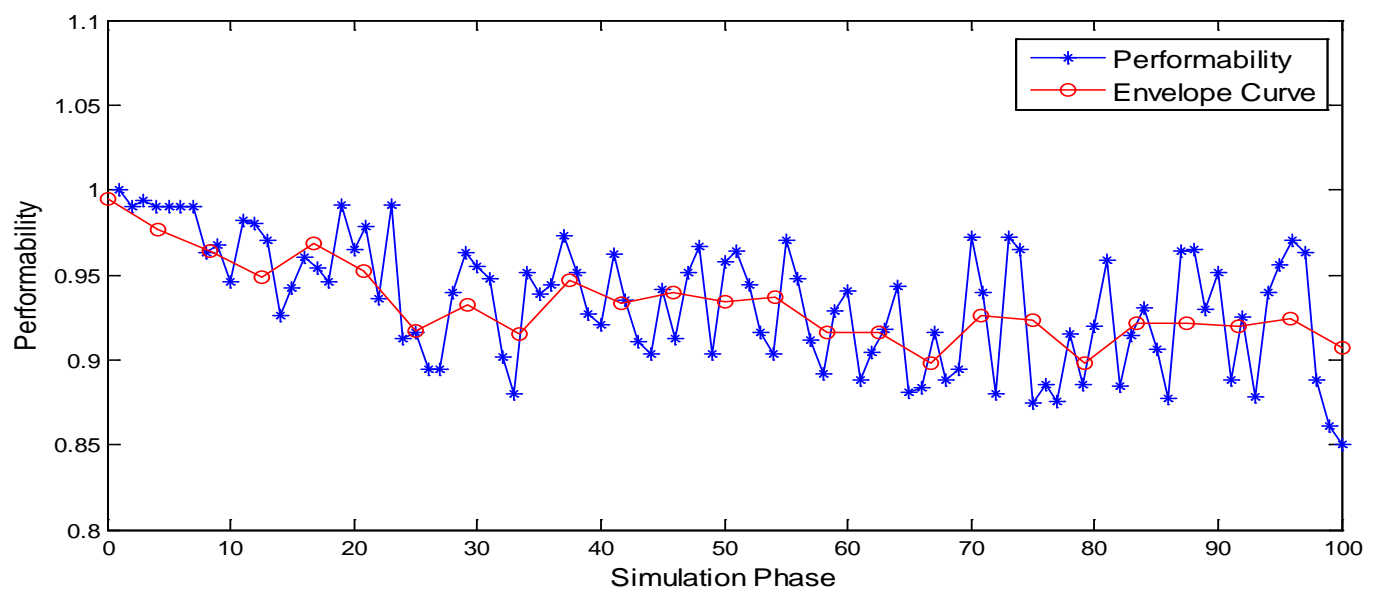

Fig. 4. The assessment result of the case.

Fig. 4 shows that the performability of the network decreases in fluctuation. The performance degradation of the nodes determines the decline in network performability and dynamic topology brings about the fluctuation.

\subsection{Modified the Propagation Model in Upper Model}

In MANETs, the objective of routing protocol in MANETs is to establish a well-organized route between transmitting and receiving nodes to send their messages [29]. And the node mobility model and the radio propagation model have strong impact on the performance of mobile wireless networks, e.g., the performance of routing protocols varies with these models. In the above scenarios, all the nodes follow the simple propagation model and mobility model.

In the above case, the nodes follow the simple and idealized propagation model in the message dissemination process. It only assumes that there is a direct path between the transmitter and receiver. The radio propagation models is a free line of sight communication between the nodes assuming obstacle free area. As a result the transmission range is nothing but a circle assuming that the nodes residing within this circle receive thetransmitted message without errors[30], as seen in Fig. 1. If the calculated range between two nodes is less than the node transmission distance, this two nodes are considered to be connected. And the link reliability between the two nodes is 1 , but in the real MANETs, after a distance radio connection is deteriorated due to the weather conditions, existence of highrise buildings or hills [31]. Thus the link reliability is less than 1 and some how decrease as the distance between the two nodes increases. We know that the reliability of link have more than two conditions ( 0 and 1$)$. Taking the reliability of link into consideration, the paths between the transmitter and receiver may change compared with the previous propagation model. Then this will affect the network performance ultimately. 
In order to modeling the reliability of link for a more realistic situation, the FS-TRG propagation model (a combination of free-space propagation model (FS) and two-ray ground propagation model) is introduced to modeling the link reliability in the upper level of the hierarchical assessment method.

In the FS-TRG model, the FS model is used at small distance, while the TRG (two ray ground propagation) model is used at larger nodal distances [31]. The reliability of links can be modeled by (8).

$$
R_{l} \begin{cases}1 & r_{i j}<d_{1} r \\ \frac{\left(d_{1}\right)^{2}}{1-\left(d_{1}\right)^{2}}\left(\frac{r^{2}}{r_{i j}^{2}}-1\right) & d_{1} r \leq r_{i j} \leq d_{2} r \\ \frac{\left(d_{1}\right)^{2}\left(d_{2}\right)^{2}}{\left(1-\left(d_{1}\right)^{2}\right)\left(1+\left(d_{2}\right)^{2}\right)}\left(\frac{r^{4}}{r_{i j}^{4}}-1\right) & d_{2} r \leq r_{i j} \leq r \\ 0 & r_{i j} \geq r\end{cases}
$$

Where $0<d_{1}, d_{2}<1, r_{i j}$ is the actual distance between node $i$ and node $j$, and $r$ is the maximum propagation of the nodes in the MANETs. Fig. 5 shows the link reliability model based on FS-TRG propagation in this case, where $d_{1}$ is 0.18 and $d_{2}$ is 0.82 .

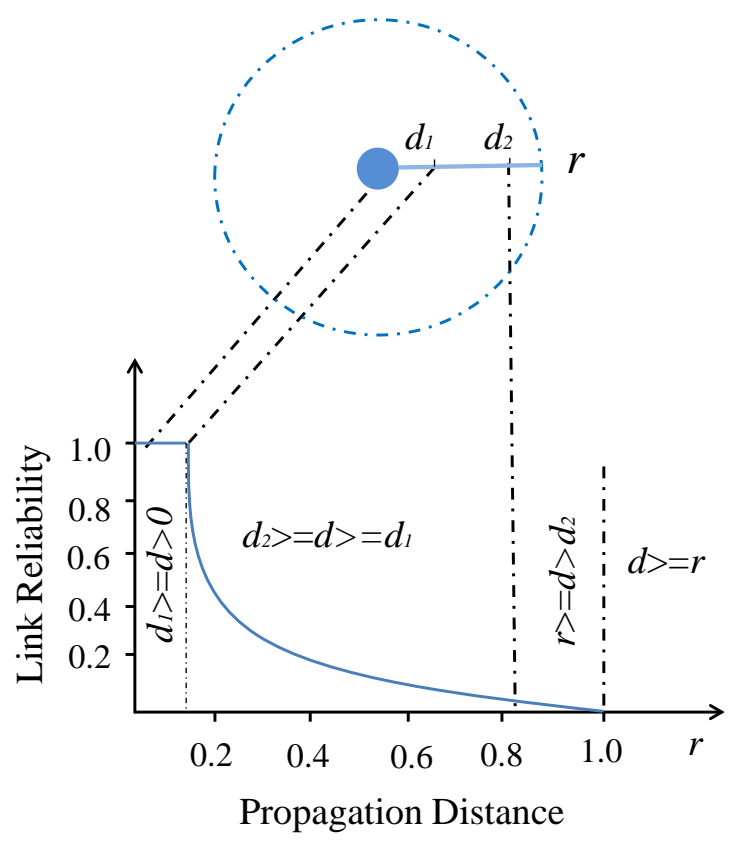

Fig. 5. The FS-TRG propagation model. 
According to the above description about the FS-TRG propagation model, the link reliability can be calculated depending on the distance between the two nodes. This propagation model is more in line with the real situation, so our hierarchical assessment method can be more reasonable when the previous simple propagation model replaced by the FS-TRG model.

Here the performability of the network can be analyzed under different propagation models.Through our hierarchical MANETs performability assessment method, we get the performability analysis result of the scenario with the FS-TRG propagation model. The assessment result comparison of the FS scenario with the FS-TRG scenario is shown in Fig. 6.

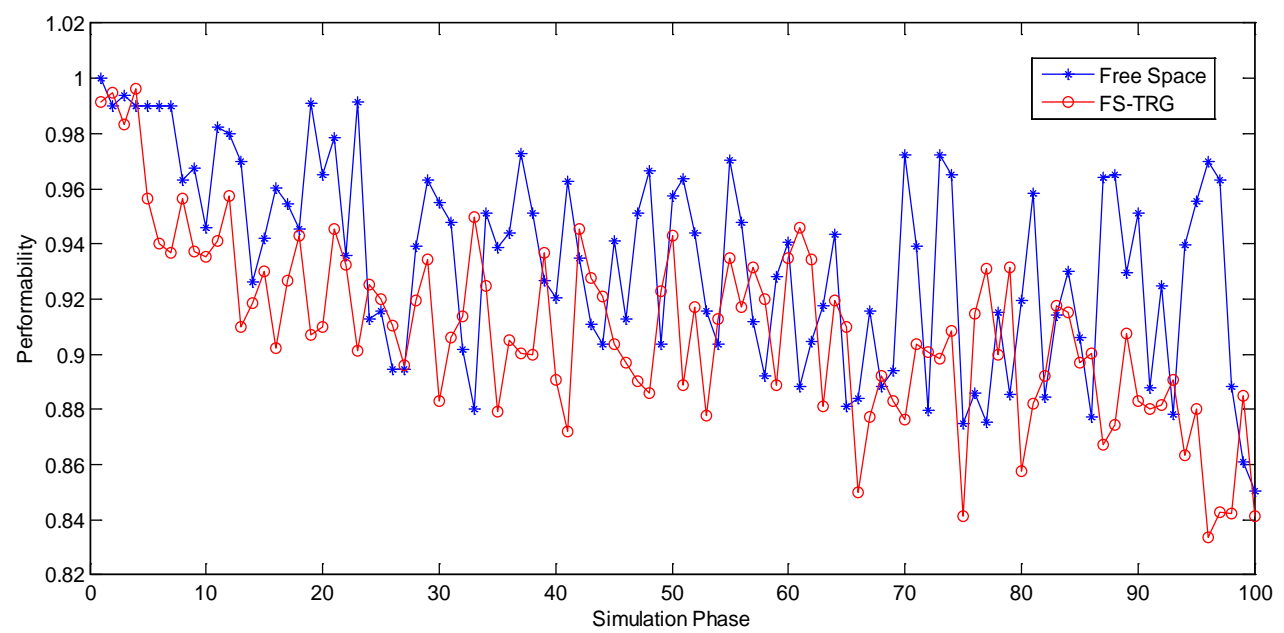

Fig. 6. The result of different propagation model.

From Fig. 6, we can observe that the performability of the two scenarios degrade similarly. However, the performability of the FS-TRG model degrades faster than the FS model. That is because the FS-TRG model take the link reliability into consideration, which means that it adds more link failures in the network, which increase the difficulty for the connectivity between nodes. In that case, the characteristic path length of the network becomes bigger, it takes more hops for a data packet to pass from the source to the terminal. Under this influence, the average delay of the network increases, and the system performability descends faster.

In general, our hierarchical performability assessment method can be improved by modifying the node propagation model in the upper level.

\subsection{Considering Different Nodes Mobility Models}

In MANETs, the node mobility model has great impact on the performance of the network, the nodes' movements becomes an important consideration for the network performability assessment. The nodes mobility model will be different when network operations under different scenarios. The random direction mobility model is a simple model to describe the 
nodes movement .We change the random direction mobility model in Section 3.1 into surround mobility. In the new mobility model, the nodes surround the coordinate of $(500,600)$ through random paths. Fig. 7 shows four distinct topologies of the network at different moments under the surround mobility.
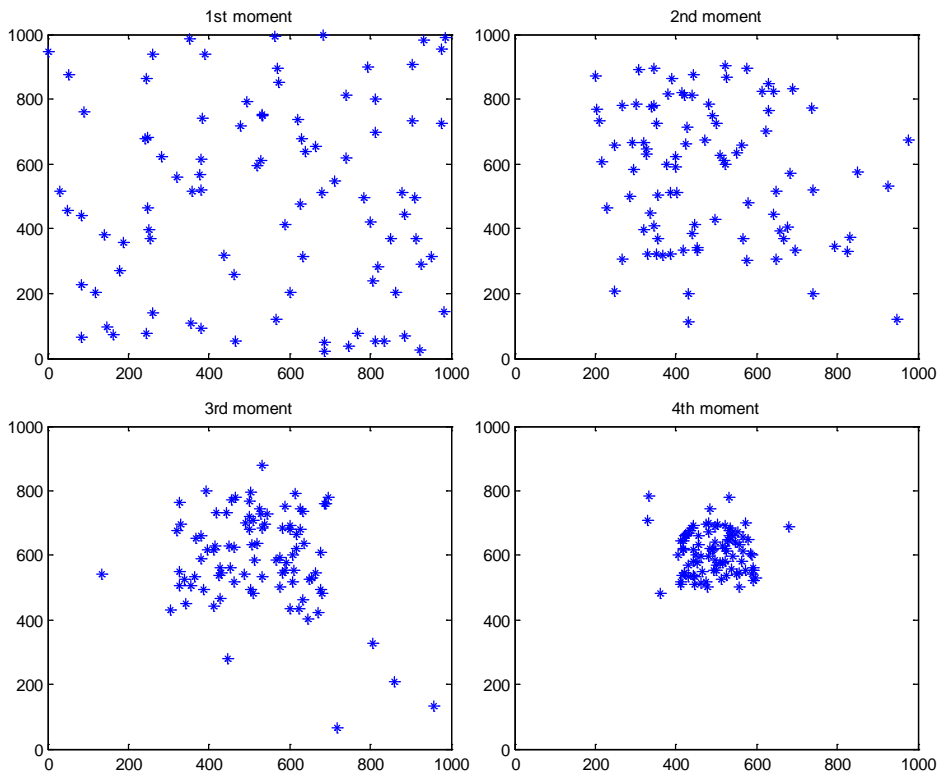

Fig. 7. Network topologies under the surround mobility.

By rewriting the mobility code in simulation program, we obtain the performability analysis result of this scenario with surround mobility. The assessment result comparison of surround mobility scenario with random direction mobility scenario is shown in Fig. 8.

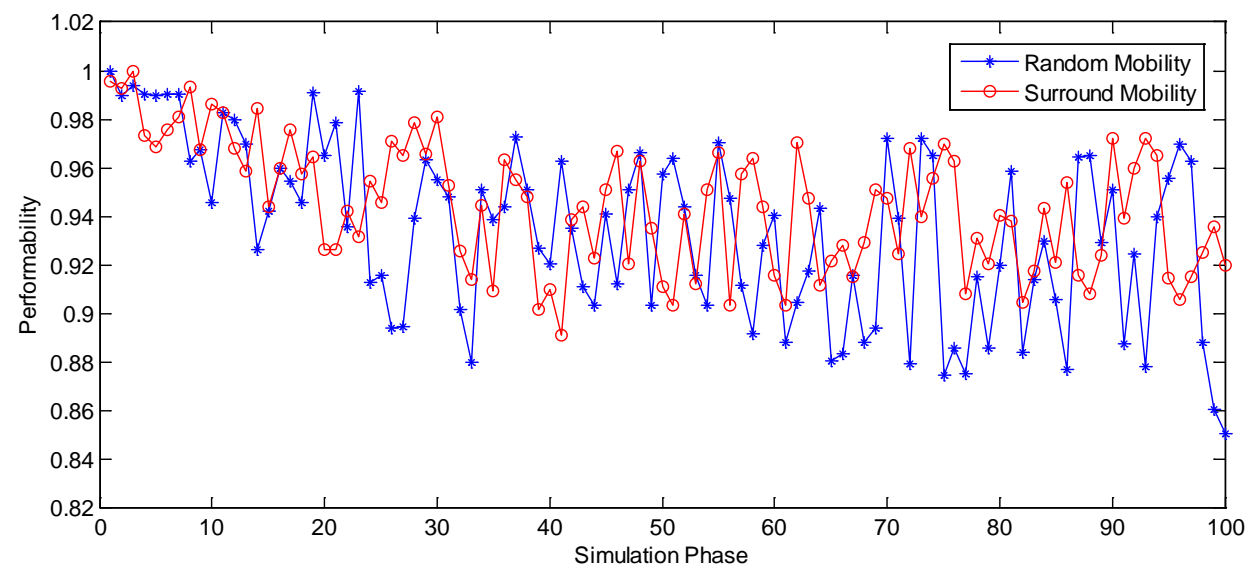

Fig. 8. The assessment result of different mobility.

From Fig. 8, it can be observed that the performability analysis results of both the two 
mobility models degrade as the time to move forward. However, the performability of the surround mobility scenario degrades slower than that of the random direction mobility scenario. That is because the mobility have an effect on the topology of the network and the topology affects the characteristic path length, which is importantly relevant to the performability of MANETs. The shorter average nodal distance in the surround mobility scenario leads to a smaller characteristic path length, which ensures a higher level of communication performance. What's more, since the network topology of the surround mobility scenario changes less frequently, its result curve has a narrower fluctuation.

\section{Validation}

In this section, the validation of our hierarchical performability assessment method is discussed. We present an accuracy verification of the model based on the comparison with OPNET simulation in Section 4.1 and then discuss the efficiency analysis of the hierarchical model by using different scale networks.

\subsection{Accuracy Verification}

In order to verify our method, a network scenario is built in OPNET based on the case in Section 3.1, as shown in Fig. 9. Then, we use the Monte Carlo simulation method to analyze the performability of this network scenario and compare the results with the analytical results in Section 3.1.

Firstly, the parameter values are set as same as those in Table 2. Secondly, the Global statistics- Wireless LAN- Delay(s) is chosen to analyze the performance of the network scenario. Finally, one thousands independent simulation experiments were accomplished to collect the delay data. After obtaining the global delay of the network through the Monte Carlo simulation, we get the performability results by a data calculating program. The results obtained from the hierarchical model and the OPNET simulations are shown in Fig. 10.

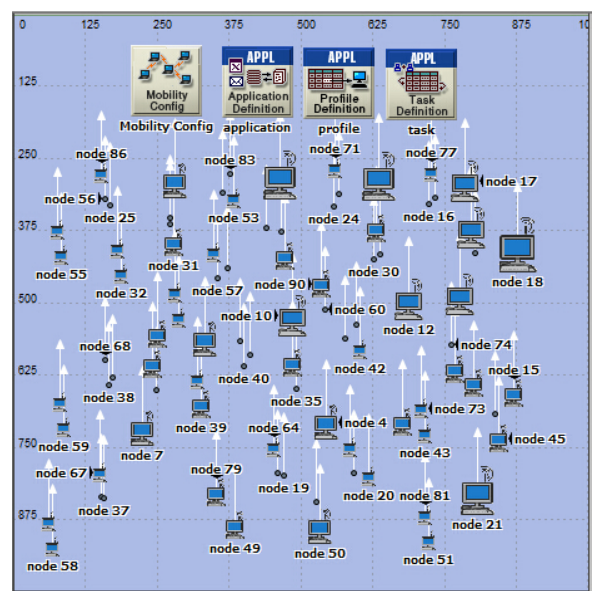

Fig. 9. The network scenario in OPNET. 


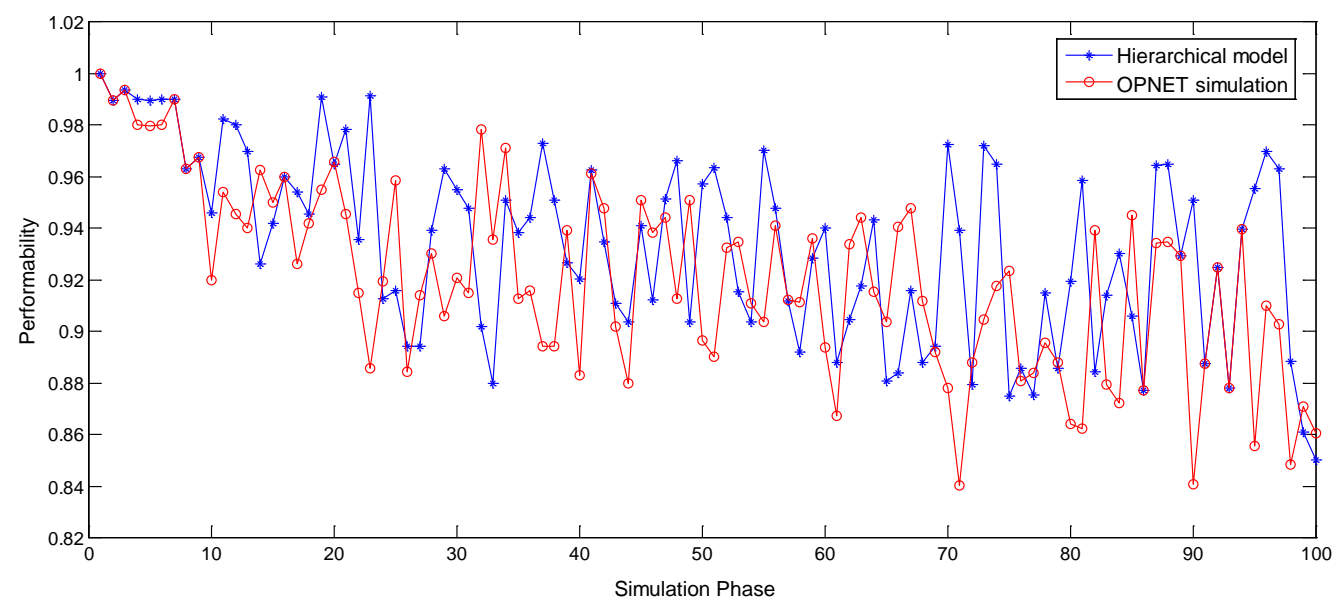

Fig. 10. Accuracy verification result.

From Fig. 10, it can be observed that the simulation results are similar with the analytical results under the proposed hierarchical MRC model. The average values of the network performability for these two methods are shown in Table 3.

It is demonstrated that the relative errors between the two methods is small. Thus, the hierarchical model proposed in this paper is feasible to analyze the performability of MANETs.

Table 3. Relative error analysis

\begin{tabular}{|c|c|}
\hline & $\begin{array}{c}\text { Average value } \\
\text { (Performability) }\end{array}$ \\
\hline Hierarchical method & 0.9339 \\
\hline OPNET simulation & 0.9203 \\
\hline Relative Error & $1.46 \%$ \\
\hline
\end{tabular}

\subsection{Efficiency Analysis}

A comparative experiment is implemented to analyze the efficiency of the assessment method based on hierarchical model. We conduct the experiments on a ThinkCentre M6490t computer, which has 4GB RAM and intel-i5 CPU. The results of the experiment are shown in Table 4 and the efficiency comparison of the two methods is shown in Fig. 11.

Table 4. Efficiency analysis result.

\begin{tabular}{|c|c|c|}
\hline $\mathrm{n}$ & OPNET simulation & Hierarchical method \\
\hline 4 & 3 seconds & 20 milliseconds \\
\hline
\end{tabular}




\begin{tabular}{|c|c|c|}
\hline 16 & 10 minutes & 600 milliseconds \\
\hline 64 & 2.5 hours & 14 seconds \\
\hline 216 & 3 days & 2.2 minutes \\
\hline
\end{tabular}

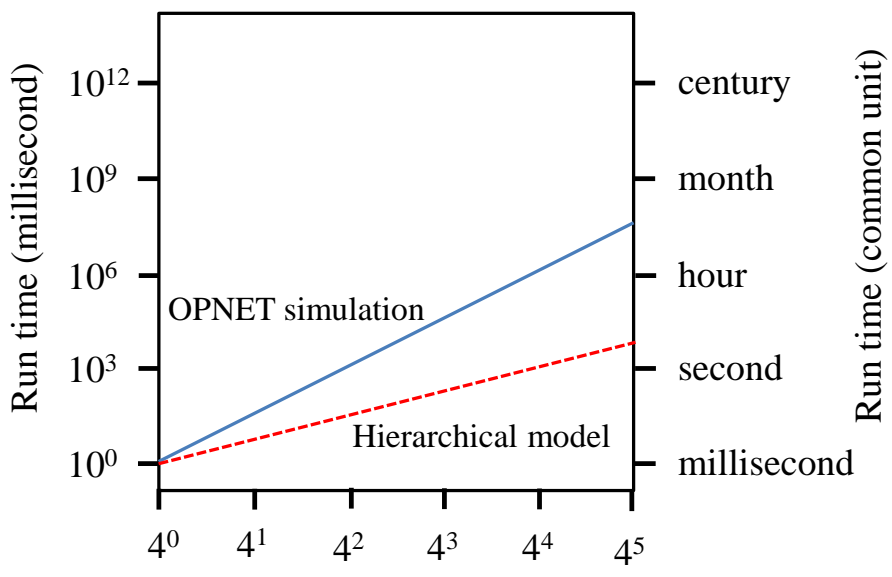

Scale of the nodes (n)

Fig. 11. Efficiency comparison result.

From Fig. 11, we can see that the hierarchical model proposed in this paper is more effective than the OPNET simulations. With the increasing scale of the network, the advantages become more and more obvious. On account of the traditional limit of OPNET, this novel method is more suitable for analyzing large-scale networks.

\section{Conclusion}

In this paper, we proposed a hierarchical model for performability assessment of large-scale MANETs. By calculating the characteristic path length of the network, we solve the state explosion problem in traditional network performability methods from the perspective of complex network. Since the lower level of the model is constructed by Markov reward chains, it can be able to take the multi-states of nodes performance into consideration, which is always ignored in complex network theory but very important to the performability of MANETs. What's more, for a more realistic situation, our hierarchical assessment method can be improved by modifying the propagation model in upper model. Also the performability of the network is analyzed under two different nodes mobility models. At last, the contrast test showed that it has better efficiency than OPNET simulation method. The advantage is more apparent as the scale of the network become larger. 


\section{References}

[1] K. S. Trivedi, E. C. Andrade and F. Machida, "Combining Performance and Availability Analysis in Practice,” Advances in Computers, vol. 84, pp. 1-38, 2012.

Article (CrossRef Link).

[2] M. Conti, E. Gregori, and G. Maselli, "Performability in ad hoc networks," in Proc. of IEEE 59th Vehicular Technology Conference, pp. 2106-2110, 2004. Article (CrossRef Link).

[3] S. W. Kim and B.-S. Kim, "OFDMA-based reliable multicast MAC protocol for wireless ad-hoc networks,” ETRI Journal, vol. 31, no. 1, pp. 83-85, 2009. Article (CrossRef Link).

[4] W. Q Wu, N Huang, and Y Zhang, "A new hybrid traffic generation model for Tactical Internet reliability test," in Proc. of 2015 Annual Reliability and Maintainability Symposium), pp. 1-6, 2015. Article (CrossRef Link).

[5] S. Distefano and A. Puliafito, "Dependability evaluation with dynamic reliability block diagrams and dynamic fault trees," IEEE Transactions on Dependable and Secure Computing, vol. 6, no. 1, pp. 4-17, 2009. Article (CrossRef Link).

[6] G. Manno, F. Chiacchio and L. Compagno, "MatCarloRe: An integrated FT and Monte Carlo Simulink tool for the reliability assessment of dynamic fault tree," Expert Systems with Applications, vol. 39, no. 12, pp. 10334-10342, 2012. Article (CrossRef Link).

[7] A. H. Azni, R. Ahmad, Z. A. M. Noh, A. S. H. Basari and H. Burairah, "Correlated node behavior model based on semi Markov process for MANETS," arXiv preprint arXiv:1203.4319, 2012. Article (CrossRef Link).

[8] M. Conti, E. Gregori and G. Maaelli, "Improving the performability of data transfer in mobile ad hoc networks," SECON : 153-163, 2005. Article (CrossRef Link).

[9] R. Groenevelt, P. Nain and G. Koole, "The message delay in mobile ad hoc networks," Performance Evaluation, vol. 62, no. 1, pp. 210-228, 2005. Article (CrossRef Link).

[10] C. Boldrini, M. Conti, and A. Passarella, "Performance modelling of opportunistic forwarding under heterogenous mobility,” Computer Communications, vol. 48, pp. 56-70, 2014. Article (CrossRef Link).

[11] M. Cello, G. Gencco and M. Marchese, "Evaluation of the Average Packet Delivery Delay in Highly-Disrupted Networks: The DTN and IP-like Protocol Cases,” Communications Letters, IEEE, vol. 18, no. 3, pp. 519-522, 2014. Article (CrossRef Link).

[12] A. Helmy, "Small worlds in wireless networks," Communications Letters, IEEE, vol. 7, no. 10, pp. 490-492, 2003. Article (CrossRef Link).

[13] A. Banerjee, R. Agarwal and V. Gauthier, "A self-organization framework for wireless ad hoc networks as small worlds," IEEE Transactions on Vehicular Technology, vol. 61, no. 6, pp. 2659-2673, 2012. Article (CrossRef Link).

[14] T. Hossmann, T. Spyropoulos, and F. Legendre, "Social network analysis of human mobility and implications for DTN performance analysis and mobility modeling," Computer Engineering and Networks Laboratory ETH Zurich, Tech. Rep, vol. 323, 2010. Article (CrossRef Link).

[15] A. Panisson, A. Barrat, C. Cattuto and B. D. Van, "On the dynamics of human proximity for data diffusion in ad-hoc networks,” Ad Hoc Networks, vol. 10, no. 8, pp. 1532-1543, 2012. Article (CrossRef Link).

[16] C. Rezende, A. Boukerche, R. W. Pazzi and P. S. Bruno, "The impact of mobility on mobile ad hoc networks through the perspective of complex networks," Journal of Parallel and Distributed Computing, vol. 71, no. 9, pp. 1189-1200, 2011. Article (CrossRef Link). 
[17] C. Tachtatzis and D. Harle, "Performance evaluation of multi-path and single-path routing protocols for mobile ad-hoc networks," International Symposium on Performance Evaluation of Computer and Telecommunication Systems, pp. 173-180, 2008. Article (CrossRef Link).

[18] N. P. Bobade and N. N. Mhala, "Performance Evaluation of Ad Hoc On Demand Distance Vector in MANETs with varying Network Size using NS-2 Simulation,” International Journal on Computer Science and Engineering, vol. 2, no. 8, pp. 2731-2735, 2010. Article (CrossRef Link).

[19] M. Saad, M. Izuan, and Z. Ahmad Zulkarnain, "Performance analysis of random-based mobility models in MANET routing protocol,” European Journal of Scientific Research, vol. 32, no. 4, pp. 444-454, 2009. Article (CrossRef Link).

[20] R. Kaur and G. S. Josan, "Performance Evaluation Of Congestion Control Tcp Variants In Vanet Using Omnet+,” Performance Evaluation, vol. 2, no. 5, pp. 1682-1688, 2012. Article (CrossRef Link).

[21] P. Garg and A. Tuteja, "Comparative Performance Analysis of Two Ad-hoc Routing Protocols," in Proc. of International Conference on Network and Electronics Engineering IPCSIT, pp. 99-104, 2011. Article (CrossRef Link).

[22] M. Rajput, P. Khatri, A. Shastri and K. Solanki, "Comparison of ad-hoc reactive routing protocols using opnet modeler," in Proc. of International Conference on Computer Information Systems and Industrial Management Applications (CISIM), pp. 530-534, 2010. Article (CrossRef Link).

[23] R. Jain, R. Kumawat, S. Mandliya, and M. Patidar, "Performance Evaluation of Table Driven Multipath Routing Protocols in MANET under varying Nodes, Traffic load \& Pause time," Performance Evaluation, vol. 2, no. 2, 2014. Article (CrossRef Link).

[24] D. Y. Chen, S. Garg and K. S. Trivedi, "Network survivability performance evaluation: a quantitative approach with applications in wireless ad-hoc networks," in Proc. of the 5th ACM international workshop on Modeling analysis and simulation of wireless and mobile systems, pp. 61-68, 2002. Article (CrossRef Link).

[25] S. Kaur, R. K. Bansal, and S. Bansal, "Performance Analysis of AODV, DSR and OLSR Routing Techniques for Ad hoc Mobile Networks,” International Journal of Computer Science Engineering and Information Technology Research, vol. 3, no. 5, pp. 195-200, 2013. Article (CrossRef Link).

[26] A. Ravinder, A. B Ahuja, and P. Ahuja, "Performance evaluation and comparison of AODV and DSR routing protocols in MANETs under wormhole attack," in Proc. of IEEE Second International Conference on Image Information Processing, pp. 699-702, 2013. Article (CrossRef Link).

[27] D. Wang and K. S. Trivedi, "Reliability analysis of phased-mission system with independent component repairs," IEEE Transactions on Reliability, vol. 56, no. 3, pp. 540-551, 2007. Article (CrossRef Link).

[28] T. Baugé, Ad Hoc Networking in Military Scenarios, Thales Research and Technology (UK), 2004.

[29] K. Zaman, M. Shafiq, J. G. Choi, and M. Iqbal, "The Life Cycle of Routing in Mobile Ad Hoc Networks: A Survey,” Smart Computing Review, vol. 5, pp. 135-149, 2015. Article (CrossRef Link).

[30] M. Siraj and S. Kanrar, "Performance of Modeling wireless networks in realistic environment," arXiv preprint arXiv:1201.0842, 2012. Article (CrossRef Link). 
[31] A. Peiravi and H. T. Kheibari, "Fast estimation of network reliability using modified Manhattan distance in mobile wireless networks,” Journal of Applied Sciences, vol. 8, no. 23, pp. 4303-4311, 2008. Article (CrossRef Link).

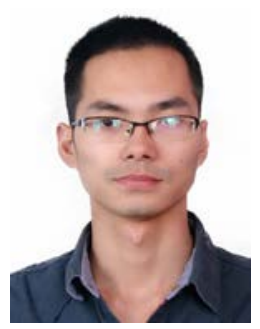

Shuo Zhang is a Master Candidate in the School of Reliability and Systems engineering, Beihang University, P.R. China. He received his B.S. degree from Harbin Engineering University, China, 2013. Now his research interests include network reliability, Ad Hoc network and complex network. E-mail: zhangshuolt@163.com.

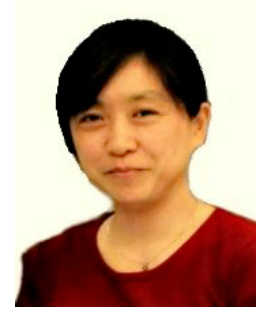

Ning Huang was born in 1968. She is a professor in the School of Reliability and Systems engineering at Beihang University, China. She obtained her Ph.D. degree in 1997 from Beihang University. She was a visiting scholar in the University of Illinois Urbana-Champaign from 2007 to 2008. She is the author of more than forty scholarly papers covering various areas of network reliability and software reliability. E-mail: hn@buaa.edu.cn

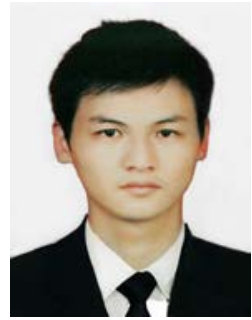

Xiaolei Sun is a Master Candidate in the School of Reliability and Systems engineering at Beihang University, China. He conducts research on network reliability, Ad Hoc network, the reliability of complex networks and propagation of network failures. E-mail: cny258sxl@163.com

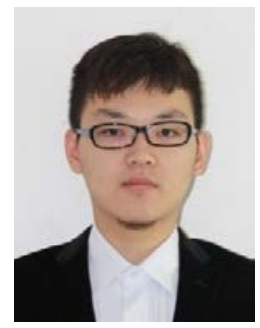

Yue Zhang received the BS degree in the School of Mathematics and Systems Science at Beihang University in 2012. Now he is pursuing a Ph.D. in the School of Reliability and Systems Engineering in Beihang University since 2013, China. His current research interests include network reliability and network traffic. E-mail: zybuaa2013@163.com 